\title{
NECESIDAD DE POLÍTICAS DE POBLACIÓN EN AMÉRICA LATINA*
}

\author{
Eduardo E. Arriaga \\ International Population and Urban Research \\ $\mathrm{y}$ \\ Departamento de Demografía \\ Universidad de California, Berkeley
}

\begin{abstract}
Aunque la mayoría de los países de América Latina no han tenido una política explícita de población, en todos los países del área se han tomado medidas que han afectado significativamente el proceso demográfico de estas poblaciones. Mientras casi nada se ha intentado en relación con la fecundidad, se adoptaron ciertos programas de salud pública que han producido un descenso rapidísimo de la mortalidad. Este hecho creó un desequilibrio demográfico que produjo una situación poco favorable para el desarrollo económico y social de estos países.

El propósito de este artículo es describir y establecer el efecto que las tendencias demográficas - fecundidad y mortalidad - han producido en estas sociedades, y analizar ciertos aspectos de la evolución de las mismas. Se verá también cómo las condiciones demográficas han interferido en la evolución de la mayoría de estos países y cómo la situación podría mejorarse si se adoptaran, para cada caso, ciertos programas tendientes a modificar la variables demográficas. Sin embargo, es necesario dejar establecido desde el comienzo de este estudio que sólo se consideran los efectos producidos por las características demográficas, sin tener en cuenta otros factores - económicos, políticos y sociales- que también afectan el comportamiento socioeconómico de estas sociedades. En otras palabras, el estudio se propone mostrar cómo las características demográficas afectan ciertas situaciones socioeconómicas de estos países, tales como crecimiento de las ciudades y problemas de transporte, desempleo y octupación disfrazada, costo de la educación y estructura de edades de la población votante e implicaciones políticas.

Pero antes de entrar a considerar cada uno de estos aspectos mencionados, será útil repasar brevemente cuáles han sido las características demográficas de América Latina.
\end{abstract}

* El autor agradece su apoyo a aquellas instituciones que prestaron ayuda financiera. Este artículo se realizó gracias a los fondos dados al International Population and Urban Research por el National Institute of Child Health and Human Development (5 R01 HD04602-03) y por el Institute of International Studies de la Universidad de California (Berkeley) de su propio fondo recibido de la Fundación Ford; y por los fondos de adiestramiento recibidos por el Departamento de Demografía de la Universidad de California provenientes de la Fundación Ford. 


\section{CARACTERÍsticas demográficas}

A juzgar por las estimaciones históricas existentes, los países de América Latina presentaban niveles altos de mortalidad y fecundidad durante el siglo pasado. A partir de los últimos decenios del siglo XIX, comenzaron a producirse cambios demográficos que se acentuaron mucho más durante el presente siglo. ${ }^{1}$ Dichos cambios se describen a continuación, comenzando con mortalidad y siguiendo con fecundidad.

Mortalidad. Argentina y quizás Uruguay fueron los países de América Latina que presentaban en 1900 los niveles más bajos de mortalidad, posiblemente con esperanzas de vida al nacimiento que variaban entre 35 y 40 años. $^{2}$ A partir del comienzo del siglo, varios otros países experimentaron un descenso paulatino de la mortalidad. Pero no fue sino desde 1930 cuando todos los países del área experimentaron un descenso rápido de la mortalidad. Sin duda que entre los factores causales de dicho descenso deben mencionarse una mejor situación económica y nutritiva de la población y, principalmente, la adopción de programas de salud tendientes a combatir las enfermedades infecto-contagiosas en cada nación. ${ }^{3}$

Fue así como en 1960 casi todos los países del área tenían una esperanza de vida al nacimiento mayor de 50 años, varios de ellos presentaban un nivel sobre 55 años, y unos pocos habían sobrepasado los 60 años de esperanza de vida. Dicho descenso tan rápido de la mortalidad se debió principalmente a las posibilidades que estos países tuvieron de "importar" medicinas, y nuevas técnicas y equipos médicos, complementado por el deseo innato del ser humano de conservar la vida durante el mayor número de años posible. Fue así como las poblaciones latinoamericanas rápidamente adaptaron y desearon utilizar todos los procedimientos y beneficios sanitarios que cada país ofreció (ver los niveles de mortalidad en el cuadro 1).

Fecundidad. Contrariamente a lo ocurrido con la mortalidad, la fecundidad permaneció a un alto nivel, y casi invariable en la mayoría de estos países. ${ }^{4}$ (Hubo excepciones como el caso de Argentina y Uruguay desde fines del siglo pasado, posteriormente Cuba, más reciente-

1 Eduardo Arriaga, New Life Tables for Latin American Populations in the Nineteenth and Twentieth Centuries, Population Monograph Series, Núm. 3, International Population and Urban Research, Institute of International Studies, University of California, Berkeley, 1968.

2 Jorge Somoza, La mortalidad de la República Argentina según tablas de vida de 1914, 1946-68 y 1959-61, CELADE, Serie A, Núm. 37, Santiago, Chile; Alberto Cataldi, Determinación de la composición por sexo y edad de la población de Uruguay, 1953, CELADE, B.60,1/5, Santiago, Chile.

3 George Stolnitz, "A Century of International Mortality Trends: I", Population Studies, 1955, Vol. 9, pp. 24-55; Kingsley Davis, "Amazing Decline of Mortality in Underdeveloped Areas", The American Economic Review, 1956, Vol. 46, pp. 305318; Eduardo Arriaga y Kingsley Davis, "The Pattern of Mortality Change in Latin America", Demography, 1969, Vol. 6, Núm. 3, pp. 223-242.

4 Andrew Collver, Birth Rates in Latin America, Research Monograph, Serie Núm. 7, International Population and Urban Research, Institute of International Studies, University of California, Berkeley 1965. 
Cuadro 1

Tendencia dE LA Mortalidad en AMÉRICA LATiNA, 1860-1970

\begin{tabular}{|c|c|c|c|}
\hline \multirow{2}{*}{$\Delta \tilde{\mathbf{n}} \mathbf{0}$} & \multicolumn{3}{|c|}{ Eaperanza de vida al nacimionto } \\
\hline & Promedio & Máximo & Mintmo \\
\hline $\begin{array}{l}1860 \\
1870 \\
1880 \\
1890 \\
1990 \\
1910 \\
1920 \\
1930 \\
1940 \\
1950 \\
1960 \\
1970\end{array}$ & $\begin{array}{l}24.4 \\
25.0 \\
25.5 \\
26.1 \\
27.2 \\
28.9 \\
31.9 \\
33.6 \\
38.0 \\
46.4 \\
55.8 \\
63.0\end{array}$ & $\begin{array}{c}\star \\
\pm \\
\pm \\
30.1 \\
31.6 \\
32.6 \\
36.8 \\
41.9 \\
48.7 \\
55.5 \\
62.2 \\
\star\end{array}$ & $\begin{array}{c}1 \\
1 \\
2 \\
23.5 \\
24.0 \\
24.6 \\
25.5 \\
26.6 \\
30.4 \\
40.7 \\
48.5 \\
\Sigma\end{array}$ \\
\hline
\end{tabular}

Fuente: Eduardo Arriaga, New Life Tables for Latin American Populations..., op. cit.

Nota: Argentina, Uruguay y Cuba no fueron incluidos en este cuadro. Si se hubiera incluido, el nivel del promedio y del máximo habrían sido más altos. Los países incluídos fueron Brasil, Bolivia, Chile, Colombia, Costa Rica, El Salvador, Guatemala, Honduras, México, Nicaragua, Panamá, Paraguay, Perú, República Dominicana y Venezuela.

mente Chile, y durante el último decenio Costa Rica y posiblemente Brasil.) Sin embargo, es necesario hacer notar que la mayoría de los países acusaron un leve aumento de la tasa bruta de natalidad entre 1940 a 1960 (véase el cuadro 2).$^{5}$ Además, se debe destacar que si bien países como Chile y Costa Rica han acusado un descenso de la fecundidad, dicho descenso no ha afectado aún significativamente la estructura demográfica de estas poblaciones, principalmente con respecto a la fuerza de trabajo. Por ello, el análisis y las conclusiones que se presentan aquí son aplicables a todos los países del área con la excepción de Argentina, Uruguay y Cuba.

La fecundidad, contrariamente a la mortalidad, no descendió, principalmente porque las actitudes humanas en relación a estos dos aspectos de la vida son completamente distintas. Mientras existe una actitud casi unánime e instintiva hacia la conservación de la vida, se presentan actitudes completamente diferentes con respecto a la fecundidad. El descenso de ésta depende de aspectos personales, culturales y sociales tanto como de los medios para controlarla. Nadie debería haberse extrañado de la tendencia de la fecundidad en América Latina. La fecundidad no pudo haber descendido dado que las condiciones socioeconómicas del área no crearon los motivos ni los deseos de la población para reducir la fecundidad. Inclusive faltó el deseo por parte de los dirigentes nacionales para provocarlas o promoverlas. No sólo la situación cultural y de desarrollo

5 Andrew Collver, op. cit.; Eduardo Arriaga, Mortality Decline and Its Demographic Effects in Latin America, Population Monograph No. 6, International Population and Urban Research, Institute of International Studies, University of California, Berkeley, 1970, capítulo VII. 
Cuadro 2

TENDENCIA de la fecundidad En América Latina. 1930.196n

\begin{tabular}{lccc}
\hline \multirow{2}{*}{ Porfodo } & & Tasas brutas de natalidsd & \\
(on miles) & & Miximo & Minimo \\
\cline { 2 - 4 } & Promodio & 46.5 & 37.4 \\
\hline $1930-1934$ & 42.4 & 45.7 & 37.8 \\
$1935-1939$ & 42.4 & 48.7 & 38.3 \\
$1940-1944$ & 43.6 & 50.7 & 37.0 \\
$1945-1949$ & 44.3 & 52.7 & 37.0 \\
$1950-1954$ & 45.5 & 50.6 & 37.6 \\
$1955-1959$ & 45.0 & & \\
\hline
\end{tabular}

Fuente: Andrew Collver, op. cit., Eduardo Arriaga, Mortality Decline and Its Demographic..., op. cit.

Nota: Se incluyeron los siguientes países: Brasil, Costa Rica, Chile, El Salvador, Guatemala, Honduras, México, Nicaragua, Panamá, Paraguay y Venezuela.

de estos países fue desfavorable para el descenso de la fecundidad, sino que además, hasta tiempos recientes, hubo una falta casi absoluta de consideración de los factores demográficos en el análisis y la planificación nacional. En otras palabras, ni la mayoría de la población ni los dirigentes nacionales pensaron en la posibilidad de que ciertos cambios demográficos pudieran afectar y contribuir al bienestar social.

Consecuencias de las tendencias demográficas. Las consecuencias inmediatas del descenso rápido de la mortalidad junto a un alto nivel de fecundidad fueron: a) un crecimiento rapidísimo de la población, nunca anteriormente observado en un área geográfica tan extensa; $b$ ) una estructura de edades extremadamente joven. ${ }^{6}$ Estos dos hechos crearon una situación no muy favorable para el desarrollo económico y cultural de las sociedades. En general, la consecuencia de estos hechos demográficos fue que ciertos problemas sociales surgieran más rápidamente de lo que habría ocurrido en otras circunstancias. Es decir, al parecer el período de tiempo transcurrido para solucionar los problemas ocasionados por cambios estructurales es más corto en América Latina de lo que fue en los países europeos durante etapas similares de evolución social.

\section{Crecimiento de la población de las ciudades}

Una idea de lo rápido que crece la población de las ciudades de América Latina se obtiene comparando a éstas con las del resto del mundo. Por ejemplo, es interesante observar la representación de ciudades latinoamericanas entre las doce ciudades mayores del mundo durante los últimos veinte años. En 1950, sólo tuna ciudad de América Latina, Buenos Aires, estaba comprendida entre las doces ciudades mayores. Diez años después, otra ciudad (Ciudad de México ) se había agregado a ellas. Recientemente,

6 Eduardo Arriaga, "The Nature and Effects of Latin American's Non-Western Trend in Fertility", Demography, Nov. 1970, Vol. 7, Núm. 4, pp. 483-501. 
en 1970, el número de las ciudades de América Latina dentro de las doce más grandes del mundo aumentó a cuatro (Río de Janeiro y São Paulo y las dos mencionadas anteriormente).$^{7}$ Expresado en cifras, la representación de latinoamericanos entre la población de las doce ciudades más grandes del mundo es:

Población de LAS DOCE CIUdAdES MAYORES DEL MUNDo (Miles de habitantes)

\begin{tabular}{lccccc}
\hline Alo & $\begin{array}{c}\text { Doos } \\
\text { Ciudades }\end{array}$ & $\begin{array}{c}\text { Ciudades } \\
\text { 1atinoamericanas }\end{array}$ & Porciento & $\begin{array}{c}\text { Otras } \\
\text { ciudades }\end{array}$ & Porolonto \\
\hline 1950 & 73063 & 5213 & 7.1 & 67850 & 92.9 \\
1960 & 92069 & 1259 & 13.6 & 79510 & 86.4 \\
1970 & 115875 & 34018 & 29.4 & 81857 & 70.6 \\
\hline
\end{tabular}

Fuente: Kingsley Davis, World Urbanization, Vol. I, op. cit.

Un ejemplo más convincente es considerar las ciudades de 100000 habitantes o más. Por ejemplo, clasificando las ciudades que en 1950 tenían dicho tamaño en dos categorías, aquellas pertenecientes a América Latina y las correspondientes al resto del mundo, y siguiendo a la población perteneciente a estos dos grupos a través de los veinte años siguientes, se obtiene la siguiente situación:

Crecimiento de la población de las ciudades que en 1950 tenían 100000 O MÁs HABITANTES (Miles de habitantes)

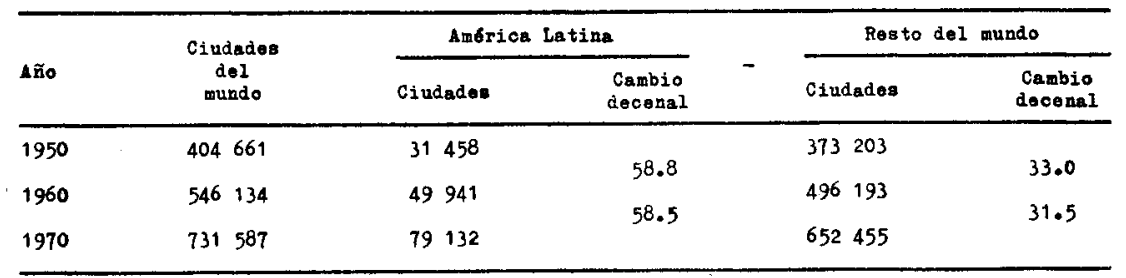

Fuente: Calculado con base en: Kingsley Davis, World Urbanization, op. cit.

Mientras la población de las ciudades de América Latina que en 1950 tenían 100000 o más habitantes aumentó 2.52 veces desde 1950 , la perteneciente a las ciudades del resto del mundo sólo aumentó 1.75 veces. Por supuesto, las ciudades del resto del mundo pertenecen a áreas completamente distintas entre sí. Sin embargo, si el mismo análisis se hace a un nivel de continente, la situación se repite. La población de las ciudades de América Latina ha registrado tasas de crecimiento más altas que la de las ciudades de cualquier otro continente (véase el cuadro 3).

Los componentes del crecimiento de las ciudades en América Latina han sido el rápido crecimiento natural de la población urbana y la inmi-

7 Kingsley Davis, World Urbanization 1950-1970, Vol. I, edición revisada, Population Monograph Series No. 4, International Population and Urban Research, Institute of International Studies, University of California, Berkeley, 1969. 
Cuadro 3

Población de las cIUdAdES QUE en 1970 tenían 100000 o Más Habitantes, POR CONTINENTES, 1950-1970

\begin{tabular}{|c|c|c|c|c|c|}
\hline & \multicolumn{3}{|c|}{ Poblaoson } & \multicolumn{2}{|c|}{$\begin{array}{c}\text { Camblo } \\
\text { porcentual }\end{array}$} \\
\hline & 1950 & 1960 & 1970 & $1950-1960$ & $1960-1970$ \\
\hline $\begin{array}{l}\text { Total mundial } \\
\text { Afrios } \\
\text { Andrica Latina g el Caribe } \\
\text { Alia } \\
\text { Akstrafia y Hueva Zolandia } \\
\text { Canad y Igtado Unidos } \\
\text { Buropa } \\
\text { U.R.S.S. }\end{array}$ & $\begin{array}{rl}404 & 661 \\
13 & 558 \\
31 & 458 \\
122 & 583 \\
5 & 019 \\
71 & 429 \\
123 & 084 \\
37 & 530\end{array}$ & $\begin{array}{rl}546 & 134 \\
19 & 736 \\
49 & 941 \\
187 & 061 \\
6 & 572 \\
93 & 621 \\
141 & 209 \\
47 & 994\end{array}$ & $\begin{array}{rl}731 & 587 \\
28 & 198 \\
79 & 132 \\
269 & 021 \\
8 & 540 \\
120 & 033 \\
165 & 380 \\
61 & 283\end{array}$ & $\begin{array}{l}35.0 \\
45.6 \\
58.8 \\
52.6 \\
30.9 \\
31.9 \\
14.7 \\
27.9\end{array}$ & $\begin{array}{l}34.0 \\
42.9 \\
58.5 \\
43.8 \\
29.9 \\
28.2 \\
17.1 \\
27.7\end{array}$ \\
\hline
\end{tabular}

Fuente: Calculado con base en Kingsley Davis, World Urbanization, Vol. I, Population Monograph Series, Núm. 4, edición revisada. International Population and Urban Research, Institute of International Studies, University of California, Berkeley.

gración hacia las mismas. ${ }^{8}$ El resultado ha sido que en general, estas ciudades han duplicado su población durante los últimos 15 años - a una tasa anual promedio de 46 habitantes por cada mil residentes. ${ }^{9}$ Si bien el crecimiento futuro es incierto, es probable que las ciudades continúen creciendo rápidamente debido a sus características demográficas. Esto produciría distintas consecuencias, entre las que sólo se consideran en este estudio aquellas directamente relacionadas con los hechos demográficos. Por ejemplo, la población de las ciudades se duplicó entre 1955 y 1970; por lo tanto, las unidades de vivienda también se deberían haber duplicado durante el mismo período. ${ }^{10}$ Es difícil determinar o conocer si esto último ocurrió.

Sin embargo, no hay duda de que la inversión requerida para satisfacer 1a demanda de viviendas no es la más aconsejable para los propósitos del desarrollo económico de estos países. En la mayoría de los casos, tanto los inversores privados como estatales no consideran que la construcción de viviendas sea una inversión de alta prioridad, salvo en aquellos casos que se desee disminuir rápidamente el desempleo. En general, las inversiones hechas en otros sectores de la economía son más lucrativas o presentan mejores perspectivas para el desarrollo económico. $\mathrm{Si}$ a la poca atracción de la construcción de viviendas por parte de los grandes inversores se agrega el hecho de que la mayoría de las nuevas familias que se forman como consecuencia del crecimiento vegetativo de las ciudades, tanto como las

8 Eduardo Arriaga, "Components of City Growth in Selected Latin American Countries", Milbank Memorial Fund Quarterly, 1968, Vol. 46, pp. 237-252; Kingsley Davis, "The Urbanization of Human Population", en Cities, Scientific American Book, Nueva York, A. Knopf, 1969.

9 Para las ciudades de 20000 o más habitantes también se encontraron tasas de crecimiento elevadas. Ver Eduardo Arriaga, "The Nature and Effects...", op. cit.

${ }_{10}$ Se desea recalcar que durante el período 1955-1970 se deberían haber construido tantas casas como las que ya existían en las ciudades en 1955, sin considerar las que se deberían haber construido por efectos de reemplazo. 
familias de los inmigrantes que llegan a las mismas, no poseen generalmente medios económicos para construir una vivienda confortable, es lógico esperar que se produzcan barrios marginales. Por lo tanto, no es extraño encontrar que una proporción considerable de la población de la mayoría de las principales ciudades de América Latina viva en barrios en los que las condiciones de vida son precarias.11

Indudablemente el contraste de las condiciones de vida entre los barrios marginales y las áreas residenciales en determinadas ciudades, es fuente de problemas sociales urbanos ya señalados por otros investigadores. ${ }^{12}$ Las ciudades son áreas relativamente pequeñas donde conviven sectores de la población con características económicas completamente distintas. Es en las urbes, donde, junto a los niveles más altos de vida del país, se encuentran niveles de subsistencia. En estas circunstancias, el descontento, el crimen y la violencia no deben extrañar a nadie.

El descontento y la violencia se acentúan aún más por la inmigración proveniente de las áreas rurales paupérrimas. Mientras los inmigrantes permanezcan en su lugar de origen, la mayoría de ellos no tiene ningún criterio de comparación en relación con condiciones de vida. Una vez llegados a las ciudades perciben que existen otros niveles económicos prácticamente prohibidos para la mayoría de ellos. Esto, probablemente, da origen a un sector de insatisfechos quienes pueden ser influenciados fácilmente por demagogos, agitadores y aun gente con buenas intenciones, quienes bajo distintos fines harán notar a este sector las diferencias de condiciones de vida existentes, provocando un clima de inquietud y desasosiego. Indudablemente las diferencias de ingresos y condiciones de vida ya existían con anterioridad a la inmigración o a la formación de los barrios marginales - ya que éstos son consecuencia de la situación económica. Es decir, si bien el problema existía con anterioridad, se encontraba en un estado pasivo o latente. Sin embargo, el crecimiento rápido de las ciudades junto con la atracción que las mismas ejercen sobre las áreas rurales, hace que dicho problema latente se transforme rápidamente en un problema real y virulento. En otras palabras, el crecimiento de las ciudades y el movimiento migratorio reducen el período de tiempo disponible necesario para solucionar problemas de distribución de ingreso. El crecimiento económico es el factor clave y responsable de esta situación. Cuanto más rápidamente crezcan las ciudades sin un bienestar económico concomitante de los sectores pobres, mayores serán las posibilidades de descontento social.

Otra de las consecuencias del crecimiento rápido de la población

11 William Mangin, "Latin American Squatter Settlements - a Problem, a Solution", Latin American, Research Review, 1967, Vol. 2, Núm. 3, pp. 65-98; I. Matos Mar, "The 'Barriadas' of Lima: an Example of Integration into Urban Life", versity Press, 1961, pp. 170-190; Andrew Pearce, "Some Characteristics of Urbanization in the City of Rio de Janeiro", en Urbanization in Latin America, pp. 191-205; Naciones Unidas, World Housing Conditions and Estimated Housing Requirements, Department of Economic and Social Afairs, Informaciones demográficas necesarias para los programas de vivienda, con especial referencia a América Latina, ST/ ECLA/CONF.9/L.12, 1962.

${ }_{12}$ Irving L. Horowitz, "Electoral Politics, Urbanization and Social Development in Latin America", en The Urban Explosion in Latin America, ed. por Glenn Beyer, Cornell University Press, 1967, pp. 215-254; Glenn Beyer, "Editor's Summary and Elaboration", en ibid., pp. 254-273. 
de las ciudades es aquella relativa a la congestión del tránsito en áreas céntricas. La mayoría de las ciudades más grandes de América Latina ha experimentado desde 1950 un aumento rápido de la circulación de vehículos por las calles céntricas. La causa principal de la congestión del tránsito ha sido desde luego el crecimiento de la población, junto con el deseo (o la necesidad) de concurrir a las áreas céntricas, y la escasez de fondos estatales para construir nuevas avenidas, viaductos $o$ subterráneos que faciliten el acceso al centro comercial de la ciudad.

Si la población de las ciudades se ha duplicado durante los últimos 15 años, se podría suponer aproximadamente que el área cubierta por las mismas también se ha duplicado. ${ }^{13}$ Sin embargo, la geometría indica que las distancias desde la periferia al centro, o cualquier otro punto dentro del área, no se duplican cuando se duplica el área sino que por el contrario crecen sólo alrededor de un $40 \%{ }^{14}$ (Desde luego esto supone que el crecimiento de las áreas de las ciudades guarda cierta forma geométrica.) Esto es, si se obtuviese un promedio de las distancias diarias recorridas por la población de una ciudad en un momento determinado y se lo comparase con el mismo promedio una vez que la población de la ciudad se haya duplicado, resultaría que la diferencia relativa entre los dos promedios sería aproximadamente del $40 \%$.

La mayoría de estas ciudades ha mantenido una sola área central donde se concentra la mayoría de las oficinas, comercios y negocios principales. En estas circunstancias, es probable que por lo menos la misma proporción de personas que vivan en la ciudad continúen viajando a las áreas céntricas durante las mismas horas del día. Como consecuencia, es probable que el total de personas que viajan desde diversas partes de la ciudad al centro de la misma también se haya duplicado durante lo últimos 15 años. Por lo tanto, las unidades de transporte que deberían pasar por las áreas céntricas durante dichas horas del día deberían haberse también duplicado. Pero este supuesto anterior puede considerarse como conservador. En realidad, debe esperarse que cuando la población de las ciudades se duplica, el número de unidades que pasa por áreas céntricas debería de crecer más del doble. Las grandes ciudades son las áreas más desarrolladas del país donde se encuentra la población con los ingresos económicos más altos. Es probable que la proporción de personas que puedan poseer un automotor aumente con el transcurso del tiempo. Por ejemplo, no sería incorrecto suponer que por lo menos tres

13 Si las nuevas áreas de la ciudad tienen la misma densidad de población anterior, el área total de la ciudad prácticamente se duplica. Sin embargo, hay dos argumentos opuestos con relación al área de la ciudad cuando su población se duplica. Podría ocurrir que las áreas nuevas de la ciudad presentaran una densidad menor que las anteriores; por lo tanto el área de la ciudad crecería más que el doble. Contrariamente, puede decirse que en las ciudades existen "espacios libres" que son "llenados" antes que los perímetros se expandan; además, sc pueden construir en las áreas anteriores grandes edificios de departamentos; ambos tenderían a concentrar más la población. Para los propósitos de este estudio, no tiene mucha importancia que el área de la ciudad crezca más o menos el doble cuando la población se duplica.

${ }_{14}$ Por ejemplo, un área circular se duplica cuando el radio se multiplica por la raíz cúbica de 2 esto es 1.4142 .. Esto realmente significa un incremento del $41.42 \%$ sobre el radio anterior. Lo mismo pasa si el área es cuadrangular. El área se duplica multiplicando la medida de los lados por el mismo factor de la raíz cúbica de 2 . 
o cuatro personas que con anterioridad utilizaban transporte público -por ejemplo, ómnibus- hayan decidido dejar tal medio de transporte y utilizar un automóvil propio con fines de ir a la zona céntrica. La deserción de sólo tres o cuatro personas por cada ómnibus (con capacidad de 30 a 50 personas) no habría afectado para nada la necesidad del transporte público. El total de unidades en este tipo de servicios se habría duplicado de todas maneras durante los 15 últimos años, pero se necesitaría espacio para los tres o cuatro automóviles adicionales. E1 supuesto de que durante 15 años sólo tres o cuatro personas por ómnibus cambiaran de actitud frente al transporte es conservador, ya que debería esperarse un mayor número de desertores durante un período de tal magnitud. ${ }^{15}$

Frente al aumento de la necesidad de unidades de transporte y por consiguiente de espacio para transitar, pocas ciudades han realizado planes $\mathrm{y}$ hechos concretos con objeto de ampliar dichas vías de acceso. En general solamente las ciudades más grandes de América Latina han tomado algunas medidas tendientes a mejorar esta situación. Caracas y Río de Janeiro han construido algunos viaductos; Buenos Aires y México han construido subterráneos; y últimamente Santiago de Chile y São Paulo han decidido también comenzar la construcción de subterráneos. En todos los casos, las medidas tendientes a facilitar el acceso a las zonas céntricas se iniciaron cuando los problemas de transporte ya eran muy graves.

Desde luego que la congestión del tránsito en las áreas céntricas no es problema de América Latina solamente, sino de todas las ciudades del mundo. Pero la característica única de las ciudades de América Latina es que dicho problema surge más rápidamente que en la mayoría de las ciudades del resto del mundo. Esta situación obedece al crecimiento tan rápido de la población de las ciudades latinoamericanas junto con la falta de capital para mejorar y/o ampliar las vías de acceso al centro de la ciudad.

\section{Fuerza de trabajo}

Las características demográficas de los países de América Latina también han afectado la fuerza de trabajo: 1) al reducir la proporción de la población en edades económicamente activas y 2) al aumentar rápidamente la población en edades económicamente activas.

La reducción de la proporción de la población en edades económicamente activas ha sido una consecuencia del cambio en la estructura por edades experimentado por la población. Las edades principalmente afectadas son las más jóvenes - de 15 a 49 años. Por ejemplo, el promedio de las distribuciones proporcionales de once países latinoamericanos que constituyen la mayor parte de la población del área fue en 1930 y 1970 el siguiente: ${ }^{16}$

15 Si bien los taxis pueden ser otra alternativa de transporte, el número de estos vehículos tendrá que aumentar mucho más que lo esperado para satisfacer la nueva demanda.

${ }^{16}$ Los países incluidos son: Brasil, Chile, Colombia, Costa Rica, El Salvador, Guatemala, Honduras, México, Nicaragua, Panamá y Venezuela. Las fuentes uti- 


\begin{tabular}{lrrr}
\hline $\begin{array}{c}\text { Grupos do } \\
\text { edad }\end{array}$ & 1930 & 1970 & Diferencia \\
\hline Total & 100.0 & 100.0 & +2.5 \\
-15 & 41.5 & 44.0 & -3.4 \\
$15-49$ & 48.7 & 45.3 & +0.2 \\
$50-64$ & 7.2 & 7.4 & +0.7 \\
$65+$ & 2.6 & 3.3 & 0 \\
\hline
\end{tabular}

Frente a un cambio de estructura de edades como el registrado, la tasa total de la fuerza de trabajo (el porciento de la población que es económicamente activa) habría tendido a disminuir si las tasas específicas por edades de actividad económica se hubieran mantenido iguales. En la realidad, las tasas específicas de actividad económica por edades han experimentado cambios entre 1930 y 1970 . Las tasas correspondientes a los menores de 20 años (ambos sexos) habrían tendido a descender como consecuencia del aumento experimentado en las tasas de asisten-

\section{Cuadro 4}

TASAS de actividad eConómica totales, para algunos píases SELECCIONADOS DE AMÉrica La'tina, 1930-1960

(Porcientos)

\begin{tabular}{|c|c|c|c|c|c|}
\hline Pain & Sexo & 1930 & 1940 & 1950 & 1960 \\
\hline Chile & $\begin{array}{l}\text { Total } \\
\text { Hombres } \\
\text { Mujeres }\end{array}$ & & $\begin{array}{l}34.7 \\
52.9 \\
16.7\end{array}$ & $\begin{array}{l}36.3 \\
55.5 \\
17.8\end{array}$ & $\begin{array}{l}32.3 \\
51.2 \\
14.2\end{array}$ \\
\hline El Salvador & $\begin{array}{l}\text { Total } \\
\text { Hombres } \\
\text { Mujeres }\end{array}$ & & & $\begin{array}{l}35.2 \\
59.3 \\
11.6\end{array}$ & $\begin{array}{l}32.1 \\
53.4 \\
11.5\end{array}$ \\
\hline Mórico & $\begin{array}{l}\text { Total } \\
\text { Hombres } \\
\text { Mujeres }\end{array}$ & $\begin{array}{r}32.3 \\
61.3 \\
4.4\end{array}$ & $\begin{array}{r}29.8 \\
56.0 \\
4.3\end{array}$ & $\begin{array}{r}32.1 \\
56.3 \\
8.6\end{array}$ & $\begin{array}{l}32.4 \\
52.8 \\
11.6\end{array}$ \\
\hline Nicaragua & $\begin{array}{l}\text { Total } \\
\text { Hombres } \\
\text { Mujeres }\end{array}$ & & & $\begin{array}{r}31.2 \\
54.5 \\
8.6\end{array}$ & $\begin{array}{l}31.6 \\
50.0 \\
12.5\end{array}$ \\
\hline Panamá & $\begin{array}{l}\text { Total } \\
\text { Hombres } \\
\text { Mujeres }\end{array}$ & & $\begin{array}{l}36.7 \\
59.1 \\
13.0\end{array}$ & $\begin{array}{l}35.1 \\
55.3 \\
14.2\end{array}$ & $\begin{array}{l}33.3 \\
51.1 \\
14.5\end{array}$ \\
\hline Venezuela & $\begin{array}{l}\text { Total } \\
\text { Hombres } \\
\text { Kujeres }\end{array}$ & & $\begin{array}{l}32.2 \\
50.4 \\
14.4\end{array}$ & $\begin{array}{l}31.8 \\
51.5 \\
11.5\end{array}$ & $\begin{array}{l}32.0 \\
51.2 \\
12.1\end{array}$ \\
\hline Venezuela (Población nativa) & $\begin{array}{l}\text { Total } \\
\text { Hombrea } \\
\text { Mujeres }\end{array}$ & & $\begin{array}{l}31.9 \\
50.0 \\
14.3\end{array}$ & $\begin{array}{l}27.2 \\
44.2 \\
10.3\end{array}$ & $\begin{array}{r}23.4 \\
37.3 \\
9.5\end{array}$ \\
\hline
\end{tabular}

Fuente: Eduardo Arriaga, Mortality Decline and Its Demographic..., op. cit., p. 140.

lizadas fueron los censos de población de cada uno de estos países para las siguientes fechas: Brasil 1920 y 1940 , Chile 1930 , Colombia 1928 y 1938, Costa Rica 1927, El Salvador 1930, Guatemala 1921 y 1940, Honduras 1930, México 1930, Nicaragua 1920 y 1930 , Panamá 1930 y Venezuela 1926 y 1936. Para 1970, la información se obtuvo de las proyecciones de población del Boletín Demográfico, Crecimiento de la Población de la Región de América Latina 1920-1980. CELADE, Año I. Vol. I, Santiago, Chile, 1968. 
cia a instituciones de enseñanza. Igualmente, las tasas para los mayores de 65 años habrían tendido también a disminuir, debido a que el proceso de urbanización permite que un mayor número de personas se acoja a los beneficios de retiro. Contrariamente, la participación de las mujeres en la fuerza de trabajo parecería haber aumentado entre las edades de 20 y 64 . Lamentablemente no hay suficientes datos históricos para conocer lo ocurrido en estos países. Además, cuando existen, la comparabilidad de los mismos puede estar afectada por cambios en la definición de "persona económicamente activa". Sin embargo, la poca información existente mostraría un descenso de la tasa de participación económica masculina y un aumento de la femenina; para ambos sexos no se observa una tendencia marcada (véase el cuadro 4). La pregunta a formular es cuántos de los clasificados como económicamente activos se encontraban desocupados o en una condición de desocupación disfrazada.

La poca información disponible al respecto indicaría la existencia de altas tasas de desempleo. Para el caso de Venezuela, la tasa de desempleo para las edades 15 a 64 años era de $6.7 \%$ para ambos sexos en 1970 (6.8 y $6.6 \%$ para hombres y mujeres, respectivamente). ${ }^{17}$ La tasa de desempleo era mucho mayor en áreas urbanas que en las rurales $(8.0 \mathrm{y}$ 3.1 , respectivamente) lo que quizá indicaría una mayor desocupación disfrazada en áreas rurales. En Chile, la tasa de desempleo ha variado considerablemente. En Santiago la tasa descendió de $7.3 \%$ en 1960 a 5.0 en 1963, para luego aumentar hasta $6.0 \%$ en $1968 .{ }^{18}$ Pero aun el desempleo es mucho más elevado en el caso de Colombia. Una información sobre Bogotá y Cali indica que la tasa de desempleo era de 11.6 y $7.5 \%$, respectivamente, para la población de 12 años y más en $1970 .{ }^{19}$ Para este país existen estimaciones de desempleo que presenta una tendencia creciente desde 9.5 a $14.6 \%$ desde 1965 a $1972 .{ }^{20}$

Las tasas mencionadas deben ser consideradas como mínimas, ya que aquellas personas que trabajaron pocas horas durante el período de referencia se han clasificado como ocupados..$^{21}$

17 Ministerio de Fomento, Dirección General de Estadística y Censos Nacionales, División de Muestreo, Encuesta de hogares por muestreo, empleo, desempleo y alfabetización, abril de 1970. Documento REH-9, Caracas, 1970.

18 Universidad de Chile, Instituto de Economía y Planificación, Ocupación y desocupación, varios números. La información también presenta datos para otras ciudades chilenas. Por ejemplo, la tasa de desempleo para algunas de ellas fue: Valparaíso y Viña del Mar (marzo de 1962) 6.5\%; Antofagasta (marzo de 1962) 8.7\%; La Serena-Coquimbo (marzo de 1962) 7.0\%; Puerto Mont (marzo de 1962) $5.6 \%$; Iquique (marzo de 1962) $7.6 \%$ y Concepción-Talcahuano (abril de 1968) $10.9 \%$

19 Departamento Administrativo Nacional de Estadística, Encuesta de hogares, Bogotá, 1971, pp. 36-37, cuadro 22.A.

${ }_{20}$ Departamento Nacional de Planeación, publicado en: Departamento Administrativo Nacional de Estadística, op. cit., p. 49.

21 Por ejemplo, en el caso de Venezuela, aquellas personas que declararon haber trabajado 5 horas por semana fueron consideradas como empleadas. Además, existe otro factor que hace aparecer los desocupados como un mínimo. Posiblemente para los entrevistados no es muy claro diferenciar entre "buscan trabajo" y "desean trabajar si tienen la posibilidad de hacerlo". En el caso de Chile, existe información para aquellos que buscan trabajo y para los que trabajarían si tuvieran la posibilidad. El número de estos últimos, clasificados fuera de la fuerza de trabajo, es tres veces mayor que el de los desocupados, y representa el $20 \%$ de la población no económicamente activa de 14 años y más. 
En relación con la información sobre la desocupación disfrazada en América Latina, no hay duda que existe mucha menos información. Sin embargo, una idea de este aspecto de la fuerza de trabajo se obtiene observando la distribución de la fuerza de trabajo entre las diversas ocupaciones. La proporción excesivamente alta de trabajadores independientes y familiares no asalariados indicaría que una parte de ellos podría considerarse como desocupación disfrazada. Aquellas personas que no teniendo ningún tipo de habilidad se encuentran desocupadas, intentan obtener algún ingreso prestando ciertos servicios que no requieren ninguna especialidad. Del mismo modo, aquellos trabajadores familiares no asalariados podrían estar bajo esta situación debido a que no encuentran ningún trabajo remunerado. En ambos casos la producción total del país sería afectada sólo ligeramente si estos trabajadores permanecieran desocupados. Una comparación internacional entre los países de América Latina junto con otros europeos muestra la posibilidad de desocupación disfrazada en estas dos categorías (véase el cuadro 5).

\section{Cuadro 5}

Porciento de la fuerza de trabajo en las CATEgorías de trabajadores INDEPENDIENTES Y TRABAJADORES FAMILIARES NO ASALARIADOS.

Diversos PAÍSES DEL MUNDO

\begin{tabular}{|c|c|c|c|c|c|}
\hline Pale & Año & Porcionto & Pafs & Año & Porciento \\
\hline $\begin{array}{l}\text { Argentina } \\
\text { Chile } \\
\text { Colowbia } \\
\text { Costa Rica } \\
\text { Ecrador } \\
\text { Honduraz } \\
\text { Mexico } \\
\text { Nicaragua } \\
\text { Panamd } \\
\text { Pere } \\
\text { Venezuela } \\
\text { Uruguay }\end{array}$ & $\begin{array}{l}1960 \\
1960 \\
1964 \\
1963 \\
1962 \\
1961 \\
1960 \\
1970 \\
1963 \\
1960 \\
1961 \\
1961 \\
1963\end{array}$ & $\begin{array}{l}14.8 \\
20.4 \\
33.2 \\
27.8 \\
47.8 \\
54.3 \\
34.7 \\
25.5 \\
41.0 \\
44.6 \\
47.8 \\
35.5 \\
15.9\end{array}$ & $\begin{array}{l}\text { Inglaterra y Gales } \\
\text { Prancia } \\
\text { Bstados Unidos } \\
\text { Suiza }\end{array}$ & $\begin{array}{l}1961 \\
1962 \\
1940 \\
1950 \\
1960 \\
1888 \\
1900 \\
1910 \\
1920 \\
1930 \\
1940 \\
1950 \\
1960\end{array}$ & $\begin{array}{r}5.2 \\
23.2 \\
21.2 \\
17.8 \\
12.3 \\
43.4 \\
39.1 \\
37.2 \\
34.2 \\
30.2 \\
28.7 \\
25.0 \\
19.0\end{array}$ \\
\hline
\end{tabular}

Fuente: Censos de población de cada país.

Pero, ¿por qué hay tanta desocupación y desocupación disfrazada, si la proporción de población en edades económicamente activas ha tendido a disminuir? La explicación se encuentra considerando los dos efectos demográficos distintos sobre la fuerza de trabajo. El descenso de la mortalidad junto con el mantenimiento de la fecundidad a niveles altos, ha originado que las estructuras de edades tiendan a ser más jóvenes, reduciendo la proporción de los que están en las edades productivas. Al mismo tiempo, las tendencias de la mortalidad y la fecundidad han producido tasas de crecimiento de la población muy elevadas. Por ejemplo, la población dentro de las edades de 15 a 64 años de los once países latinoamericanos considerados con anterioridad creció a una tasa anual media de 24 por mil durante el período 1930 a $1970-25$ por mil para la población total. Dichas tasas se refieren a un promedio de 40 años. Las tasas para el período $1960-1970$ fueron de 31 y 29 por mil para la población total y en edades activas, respectivamente. El pro- 
blema es lograr mantener la oferta de trabajo al mismo ritmo de crecimiento de la población. Como esto no es fácil, se debe esperar desocupación.

Frente a los problemas de desocupación y desocupación disfrazada, se deben analizar las posibles soluciones. Lamentablemente la respuesta no es muy promisoria, debido a las características económicas, culturales y demográficas de los países de América Latina. Demográficamente se podría lograr una reducción del desempleo o desocupación disfrazada, si se disminuyera la tasa de crecimiento de la población en las edades activas. Esto significaría una reducción de la fecundidad cuyo efecto en la población en edades activas (15-64) comenzaría sólo después de 15 años de iniciado el descenso de la fecundidad. Dicho en otra forma, aquellos que entrarían en las edades activas a partir de un momento determinado y durante los próximos 15 años ya han nacido. Por lo tanto, la población en las edades activas continuará creciendo a un ritmo similar al de la población total durante los 15 años anteriores.

Desde un punto de vista económico, el desempleo y la desocupación disfrazada podrían combatirse aumentando las inversiones - suponiendo que se dispone de capital, el cual no siempre es fácil formar dentro de cada país, ni tampoco obtener internacionalmente..$^{22}$ En este caso, quizá se presentaría el problema de decidir el tipo de inversiones que convendría realizar: con uso intensivo de mano de obra o de capital. Las primeras, si bien tenderían a reducir el desempleo, significarían una productividad por trabajador relativamente baja; por consiguiente, los salarios serían también bajos. Si bien se mejoraría la situación de aquellas personas que están desocupadas, habría una tendencia a mantener una distribución desequilibrada del ingreso familiar. Contrariamente, si se utilizan inversiones de gran densidad de capital, la desocupación no se reduciría tan rápidamente, excepto en los casos que existiera gran disponibilidad de capital. Si esta última alternativa fuera la realidad, difícilmente se dispondría de la mano de obra especializada necesaria. Las inversiones de mayor densidad de capital requieren a veces un nivel de educación (vocacional y/o especial) que sólo una pequeña parte de la población de América Latina posee. Por lo tanto, antes de realizar inversiones masivas de capital, sería necesario mejorar primero la calidaḍ de la mano de obra. Dicha calidad depende, desde luego, de los programas educacionales que el país ofrece a la población y consecuentemente se relaciona con el costo de la educación, que se considera a continuación.

\section{COSTO DE LA EDUCACIÓN}

Al igual que en el caso de la fuerza de trabajo, el costo de la educación está afectado por el crecimiento de la población y por la estructura por edades. Es obvio que si la población crece con rapidez, similarmente lo hará la población en edades escolares. De aquí que el costo total de la educación deberá crecer por lo menos tan velozmente como la población en edades escolares, si se desea que la situación edu-

22 Las garantías nacionales requeridas algunas veces por los inversionistas extranjeros no se encuentran siempre en los países, o también como suele ser, no son convenientes para el país. 
cativa no se deteriore. El razonamiento es sencillo y no merece mayores explicaciones.

Sin embargo, el efecto de la estructura por edades sobre el costo de la educación - y específicamente los cambios de la mortalidad y la fecundidad - necesita cierta explicación. En general, puede decirse que cuanto más joven sea la estructura por edades de una población, menos favorables son las condiciones para un sistema educativo.

La educación de una sociedad se paga directa o indirectamente por las unidades productivas existentes en tal sociedad: la fuerza de trabajo. Aun en casos donde la educación primaria y secundaria estatal sea gratuita, la misma se paga a través de la recolección de impuestos. Estos últimos son generalmente pagados directa o indirectamente por los consumidores (a través del precio de venta) cuyo ingreso proviene de la participación en la fuerza de trabajo. Por lo tanto, una forma de medir el costo de la educación en una sociedad es sobre la base del costo por trabajador.

Tal como vimos anteriormente, el cambio de estructura por edades de la población produjo una reducción de la población en edades productivas, mientras que la proporción de población en edades jóvenes (menores de 15 años) aumentó. Si se supone que las tasas de asistencia escolar, el costo de la educación por estudiante, la calidad de la educación y las tasas específicas de actividad económica no han cambiado, lógicamente el costo de la educación por trabajador habría aumentado en los países de América Latina.

Desde luego, ninguno de los supuestos anteriores es real, ya que todas las variables mencionadas cambian a través del tiempo. Las tasas de asistencia escolar generalmente aumentan; el costo de la educación por estudiante sigue la misma tendencia ascendente debido a una mejor calidad de la educación, mientras que la proporción de personas que trabaja tiende a descender en el caso de los hombres y a aumentar en el de las mujeres.

Los efectos que los cambios de estas variables han producido sobre el costo de la educación por trabajador han sido considerados en un estudio reciente, donde se concluye que la situación demográfica de América Latina ha creado una situación que es menos ventajosa para la evolución educativa de lo que fue la situación en otros países donde se registró un descenso de la fecundidad. ${ }^{23}$ Dicho estudio concluye que el costo de la educación en América Latina por trabajador es $50 \%$ más alto de lo que fue en Suecia o Estados Unidos al mismo nivel del proceso educativo; esto es, los países de América Latina podrían haber experimentado un costo de educación por trabajador menor al que realmente han tenido en 1970 si la fecundidad hubiera descendido durante el pasado. Por lo regular, un descenso de la mortalidad produce un aumento del costo de la educación por trabajador, mientras que el descenso de la fecundidad tiende a reducirlo. En América Latina, hasta 1960, mientras la mortalidad descendía rápidamente, la fecundidad se mantenía casi constante, aumentando en ciertos casos. ${ }^{24}$ Por lo tanto, no

23 Eduardo Arriaga, "Impact of Population Changes on the Education Cost" a ser publicado en Demography, 1972. op. cit.

\footnotetext{
4 Andrew Collver, op. cit., Eduardo Arriaga, "The Nature and Effects...",
} 
sólo la situación en América Latina con relación al costo de la educación por trabajador era más desfavorable que en países como Suecia y Estados Unidos, sino que, además, la situación tendió a ser cada vez más desfavorable. Un descenso de la fecundidad habría producido una situación mucho más favorable para alcanzar las metas educacionales.

\section{Población Electoral E IMPlicaciones políticas}

Generalmente, los cambios en las actitudes sociales se producen más bien por los nuevos enfoques de cada generación, que por cambios ocurridos dentro de las mismas generaciones. Esto es, los cambios tienden a producirse no tanto por los cambios de actitudes que ocurren dentro de las mismas generaciones, sino porque las nuevas generaciones desarrollan actitudes distintas a las de las generaciones precedentes frente a una misma realidad. La interpretación de hechos políticos, económicos y sociales, tanto como lo que la sociedad acepta como propio y moral, difiere más entre personas jóvenes y de edad avanzada que entre las personas de la misma edad. Por lo tanto, los cambios en las costumbres de vida tanto como en los pensamientos de la sociedad se ven afectados por la estructura de edades de aquella parte de la población que tiene el derecho de hacer valer su opinión, y por el tiempo requerido para renovar la mayoría de la población adulta. Los pensamientos de aquellos miembros de la sociedad que recientemente han entrado en las edades adultas - cuando ya pueden votar y por lo tanto pueden afectar las decisiones de la sociedad- diferirán de los pensamientos pertenecientes a personas de mayor edad. El nuevo enfoque hacia ciertos problemas significará generalmente un cambio más drástico al posible cambio concebido por aquellos de más edad. ${ }^{25}$

En consecuencia, el tiempo requerido para obtener una "nueva" mayoría de personas que pueda tomar decisiones debe tenerse en cuenta cuando se analizan ciertos aspectos políticos de las sociedades. Dado que más de la mitad de los países de América Latina tiene un gobierno elegido electoralmente, es interesante analizar ciertos aspectos demográficos de los votantes. ${ }^{26}$ Debido a que estas poblaciones han mantenido una fecundidad alta durante el pasado, la estructura por edades de los votantes es joven. Por ejemplo, si suponemos que la población de un país comienza a votar aproximadamente a la edad de 20 años, se puede determinar a qué edad se obtiene el $50 \%$ de la población votante (la edad mediana). Excluidas Argentina, Uruguay y Cuba, dicha edad

${ }_{25}$ En esta parte el artículo se refiere al pensamiento general de la sociedad. Indudablemente podría haber casos en que unas pocas personas de edad avanzada - una sola podría ser suficiente- podrían presentar un enfoque completamente distinto de la vida social, que más tarde podría influir a las generaciones jóvenes venideras.

26 En agosto de 1971, doce países de América Latina entre los veinte existentes, tenían un gobierno que había sido elegido electoralmente: Colombia, Costa Rica, Chile, República Dominicana, Ecuador, El Salvador, Guatemala, Honduras, México, Nicaragua, Venezuela y Uruguay. (EI caso de Ecuador puede ser discutido dado que el gobierno, aunque fue electo, no llamó a nuevas elecciones cuando correspondía). La población que estos países tenían era sólo el $46 \%$ de la población total de América Latina. La proporción de países y de población sería aún menor si los países que tuvieron elecciones sin una verdadera oposición fueran retirados de la lista. 
varía entre los 35 y los 38 años, con un promedio de 35.8 años (véase el cuadro 6). Contrariamente a América Latina, en países del tipo europeo donde la fecundidad ha descendido durante el pasado, la edad mediana de los votantes se encuentra alrededor de los 45 años (el promedio para los países presentados en el cuadro 6 es 45.4). La diferencia de la edad mediana de los votantes entre los dos grupos de países (alrededor de 9 años) tiene su significado real cuando dicha diferencia se compara con el tiempo requerido para lograr una mayoría "nueva" de votantes, esto es, la edad mediana de los votantes menos la edad a la que empiezan a votar (20 años). Mientras en América Latina se obtiene que un nuevo grupo puede dominar las elecciones cada 16 años (en promedio), para los países de Europa se necesitarían alrededor de 25 años.

\section{Cuadro 6}

EdAD MEdiana de LA POBLACIÓN DE 20 AÑOS DE EDAd O MÁS EN DIVERSOS PAÍSES

\begin{tabular}{llllll}
\hline Pala & Año & Mediana & Pais & Año & Mediana \\
\hline Argentina & 1970 & 41.5 & Panama & 1970 & 36.3 \\
Bolivia & 1970 & 35.8 & Paraguay & 1970 & 35.0 \\
Brasil & 1970 & 36.3 & Perá & 1970 & 35.6 \\
Chilo & 1970 & 37.8 & Rep. Dominicana & 1970 & 34.8 \\
Colombia & 1970 & 35.8 & Uruguay & 1970 & 42.6 \\
Costa Rica & 1970 & 35.8 & Venezuela & 1970 & 36.6 \\
Cuba & 1970 & 38.7 & & 1961 & 45.9 \\
Ecuador & 1970 & 35.7 & Alemania Occidental & 1970 & 44.5 \\
El Salvador & 1970 & 35.6 & Eatados Unidos & 1962 & 46.3 \\
Guatemala & 1970 & 36.1 & Francia & 1961 & 43.0 \\
Halti & 1970 & 36.1 & Italia & 1961 & 46.9 \\
Honduras & 1970 & 34.8 & Reino Unido & 1960 & 46.7 \\
Merico & 1970 & 35.7 & Suecia & 1960 & 44.5 \\
Nicaragua & 1970 & 35.6 & Suiza & & \\
\hline
\end{tabular}

Fuente: Calculado con base en: América Latina: CELADE, Boletín Demográfico, Año 1, Vol. I; Santiago de Chile, 1968; Estados Unidos: Censo de Población de 1970; Países Europeos: Naciones Unidas, Demographic Yearbook 1965, Nueva York, 1966.

El efecto de la estructura de edades de los votantes sobre una elección puede ser ilustrado con un ejemplo. Supongamos que existen dos países, uno con características demográficas como las de América Latina, y otro con las pertenecientes a países del tipo europeo occidental. Además, supongamos que las dos naciones presentan las siguientes similitudes: a) sólo hay dos candidatos en cada país, presentados por los dos únicos partidos políticos denominados I y II; b) cada persona de la misma edad vota por el candidato del mismo partido; c) el candidato del partido I es apoyado por los votantes más jóvenes, supongamos menores de 40 años; mientras que el candidato del partido II es apoyado por aquellos votantes mayores de 40 años de edad. Los resultados que se obtendrían de las elecciones en dos países con estas características serían: en el país con una estructura demográfica como América Latina, se elegiría al candidato del partido I, mientras que en el otro país se elegiría el candidato del partido II. Debe insistirse en que estos 
resultados se obtendrían a pesar de que en cada país las personas de la misma edad hubieran votado por el mismo partido.

Dado que la estructura por edades de los votantes en América Latina es joven, las tendencias políticas serían encaminadas hacia un enfoque más liberal, a pesar de que algunos sectores de la población - principalmente aquellos de edad más avanzada - no compartieran dicha tendencia. Chile podría indicarse como ejemplo de lo anterior si se toma el período de las tres últimas elecciones presidenciales $(1958,1964$ y 1970). Es probable que las características demográficas chilenas hayan tenido cierta influencia en los resultados electorales. Si bien este país ha presentado un descenso de la fecundidad desde varios años atrás, todavía no ha llegado a afectar significativamente la estructura por edades de la población de 20 o más años. En 1970, la edad mediana de los mismos era de 37.8 años. La estructura por edades chilena ha sido, como en la mayoría de los países de América Latina, favorable a una orientación liberal. Además, en este caso particular, deben agregarse otros factores que han podido ser muy importantes en la determinación de dicha tendencia política hasta 1970: a) la concentración de la población en ciudades, sin un rápido mejoramiento económico entre los obreros; $b$ ) la gran facilidad de la difusión de propaganda como consecuencia de la concentración urbana; c) una notable mejora del nivel educativo de la población sin una mejora concomitante de la situación económica. Desde luego, no se pretende explicar el caso chileno solamente en función de factores demográficos. Otros hechos sociales, políticos y económicos posiblemente hayan tenido más peso en relación con la tendencia política chilena. Sin embargo, no hay duda de que la estructura por edades de los votantes es un factor que puede influir considerablemente en los resultados electorales. Principalmente cuando además de una estructura joven de edades, se tiene un nivel educativo relativamente alto, sin una mejora económica paralela. En general, la estructura por edades de los votantes en la mayoría de los países de América Latina, junto con los sistemas electorales presentes y con la situación económica de estos países, son favorables para que sean electos los candidatos apoyados por la juventud, generalmente los más liberales.

\section{CONCLUSIONES}

El propósito de este estudio ha sido mostrar cómo las características de la población pueden afectar la evolución de ciertos aspectos de las sociedades. Ciertos países europeos han logrado un desarrollo económico y social que se considera como guía y deseable para la mayoría de los países que aún no han logrado dicho desarrollo. Estos países no industrializados han presentado diversos factores que han contribuido a retardar el desarrollo; uno de estos factores ha sido las características demográficas de las poblaciones. Muy pocas veces se han considerado e incluido las variables demográficas en los análisis y programas económicos de estas sociedades en desarrollo. En el caso de América Latina, su comparación con los países de Europa occidental ejemplifica plenamente cuán distintas fueron las características demográficas de 
ambas regiones al comienzo del desarrollo económico. Si bien, hace 40 años, los países de América Latina crecían lentamente tal como lo habían hecho los países europeos al comienzo del desarrollo económico, la estructura por edades era completamente distinta. En Europa la fecundidad se encontraba a un nivel más bajo que en América Latina, lo que producía una estructura por edades más vieja que en el caso de los países latinoamericanos. Los países de Europa mantuvieron tasas de crecimiento bajas, ya que la mortalidad y la fecundidad fueron descendiendo casi paralelamente una vez que comenzó el desarrollo económico. En los países de América Latina la situación fue distinta. Sólo la mortalidad descendió, lo que produjo no sólo el mantenimiento de una estructura de edades muy joven, sino además, una tasa de crecimiento de la población sumamente elevada. La situación hubiera sido bastante distinta si se hubiera producido un descenso de fecundidad paralelamente al de la mortalidad.

La población de las ciudades habría crecido más lentamente. Como consecuencia, no sólo los barrios marginales habrían contenido un menor porciento de población, sino además la congestión del tránsito automotor se habría producido más lentamente. Las personas encargadas de solucionar los problemas urbanos, de habitación, tránsito, expansión de alcantarillado, agua potable, potencial eléctrico, e inclusive el de las contaminaciones (aire, agua, tierra) habrían tenido más tiempo para estudiar y presentar las soluciones requeridas. Si la reducción de la fecundidad se hubiera acompañado con una política de urbanización y ruralización (intentando evitar grandes corrientes migratorias hacia las ciudades) la situación económica y social de la mayoría de estos países habría sido mejor.

Las condiciones de empleo también habrían sido mejores si la fecundida hubiera descendido. Ahora bien, el efecto del descenso de la fecundidad sobre la fuerza de trabajo no es inmediato. Se necesitan por lo menos quince años para afectar a la fuerza de trabajo desde el momento en que la fecundidad comience a variar. Si la fecundidad hubiera descendido varios decenios antes, la población en edades activas crecería en el presente a un ritmo más lento; y posiblemente, como consecuencia, el desempleo y la desocupación disfrazada serían menores. Además, dicho descenso de la fecundidad habría tenido el efecto de reducir la proporción de personas en las edades no productivas. Por lo tanto, la tasa total de la fuerza de trabajo habría tendido a aumentar, o lo que es lo mismo, el número de dependientes por trabajador se habría reducido. Indudablemente las condiciones habrían sido más favorables para mejorar los niveles de vida.

La reducción de la fecundidad no habría hecho disminuir el costo de la educación. Las poblaciones de América Latina aún necesitan incrementar la asistencia a los establecimientos educacionales y ampliar los programas educacionales, principalmente en la educación secundaria y vocacional. Sin embargo, la reducción de la fecundidad habría tenido el efecto de producir costos educativos menores de los que resultaron manteniendo la fecundidad a alto nivel. Un descenso de la natalidad habría significado lograr mejores programas educacionales con el mismo esfuerzo económico. 
La reducción de la fecundidad habría tenido efecto sobre la estructu ra por edades de los votantes. Proporcionalmente el número de votantes en las edades más avanzadas habría aumentado. Lo mismo habría ocurrido con el número de años necesarios para obtener una "nueva" mayoría de votantes. Desde luego, si bien este hecho podría haber af ectado la estabilidad política y económica del país no hay que olvidar que el efecto que tienen otras instituciones sociales y gubernamentales no se analiza aquí. En este trabajo solamente se presenta el hecho objetivo de que el descenso de la fecundidad aumentaría el poder electoral de las personas en edades medianas y avanzadas.

En general, un descenso de la fecundidad sería un factor beneficioso para el mejoramiento de las condiciones de vida de estas sociedades. La magnitud del descenso dependerá de las condiciones presentes en cada uno de los países, y del resultado de un minucioso estudio científico de las situaciones económicas, sociales y culturales de estas sociedades.

Indudablemente los países de América Latina necesitan programas de población. Estos programas deberán analizar cada uno de los aspectos demográficos tales como mortalidad, fecundidad, migraciones, urbanización, distribución geográfica de la población, población en edad escolar y fuerza de trabajo. Una vez analizados estos aspectos demográficos, se deberán relacionar con los fines socioeconómicos de las sociedades para evaluar las posibilidades de éxito. Estas evaluaciones darán la pauta sobre qué medidas demográficas convendría tomar a efecto de obtener una situación más favorable. Dejar que estas poblaciones continúen bajo un estado de laissez faire en todos los aspectos demográficos, menos en el de mortalidad, no es el mejor plan de acción. Los dirigentes sociales y aquellos que tienen la posibilidad de influir en las decisiones de la población tienen la responsabilidad de iniciar los estudios necesarios para determinar qué es lo más conveniente para el país en relación con las tendencias y el movimiento de la población. Una vez hecho esto, es también responsabilidad de ellos el convencer a la población de que es necesario tomar ciertas medidas demográficas para el beneficio de la sociedad. Es parte de la acción de un gobierno determinar qué cambios ayudarían a obtener mejores condiciones de vida, y facilitar a la sociedad los instrumentos necesarios para que dichas metas se logren. 
